\title{
MRPS23 amplification and gene expression in breast cancer; association with proliferation and the non-basal subtypes
}

\author{
Elise Klæstad ${ }^{1}\left[\right.$ [ $\cdot$ Signe Opdahl ${ }^{2} \cdot$ Monica Jernberg Engstrøm ${ }^{1,3} \cdot$ Borgny Ytterhus $^{1} \cdot$ Elisabeth Wik $^{4,5}$. \\ Anna Mary Bofin ${ }^{1} \cdot$ Marit Valla ${ }^{1,6}$
}

Received: 27 July 2019 / Accepted: 9 January 2020 / Published online: 16 January 2020

(c) The Author(s) 2020

\begin{abstract}
Purpose MRPS23 is recognized as a driver of proliferation in luminal breast cancer. The aims of the present study were to describe MRPS23 copy number change in breast cancer, and to assess associations between MRPS23 copy number change and molecular subtype, proliferation and prognosis, and between MRPS23 gene expression and molecular subtype and prognosis. Methods Using fluorescence in situ hybridization (FISH), we examined MRPS23 and centromere 17 copy number in 590 formalin-fixed, paraffin-embedded primary tumours and 144 corresponding lymph node metastases from a cohort of Norwegian breast cancer patients. Furthermore, we analysed MRPS23 gene expression data in 1971 primary breast cancer tumours from the METABRIC dataset. We used Pearson's $\chi^{2}$ test to assess associations between MRPS23 copy number and molecular subtype and proliferation, and between MRPS23 expression and molecular subtype. We studied prognosis by estimating hazard ratios and cumulative incidence of death from breast cancer according to MRPS 23 copy number and MRPS23 expression status.

Results We found MRPS23 amplification (mean MRPS23 copy number $\geq 6$ and/or MRPS23/chromosome 17 ratio $\geq 2$ ) in $8 \%$ of primary tumours. Copy number increase associated with non-basal subtypes and higher tumour cell proliferation (Ki67). Higher MRPS23 expression associated with the Luminal B subtype. We found no significant association between MRPS 23 amplification or MRSP23 gene expression, and prognosis.

Conclusion Amplification of MRPS23 is associated with higher proliferation and non-basal subtypes in breast cancer. High MRPS23 expression is associated with the Luminal B subtype.
\end{abstract}

Keywords MRPS23 - Breast cancer · Proliferation · Copy number · Amplification · METABRIC

Electronic supplementary material The online version of this article (https://doi.org/10.1007/s10549-020-05532-6) contains supplementary material, which is available to authorized users.

Elise Klæstad

elisekl@stud.ntnu.no

1 Department of Clinical and Molecular Medicine, Faculty of Medicine and Health Sciences, Norwegian University of Science and Technology, Erling Skjalgssons gate, 7030 Trondheim, Norway

2 Department of Public Health and Nursing, Faculty of Medicine and Health Sciences, Norwegian University of Science and Technology, Trondheim, Norway

3 Department of Breast and Endocrine Surgery, St. Olav's Hospital, Trondheim University Hospital, 7006 Trondheim, Norway
4 Department of Clinical Medicine, Section for Pathology, Centre for Cancer Biomarkers CCBIO, University of Bergen, 5021 Bergen, Norway

5 Department of Pathology, Haukeland University Hospital, 5021 Bergen, Norway

6 Department of Pathology, St. Olav's Hospital, Trondheim University Hospital, 7006 Trondheim, Norway 


\section{Introduction}

Increased proliferation is a hallmark of cancer $[1,2]$, and identification of genetic drivers of proliferation could be important for prognostication and development of new targeted treatment. By high-throughput genomic analyses, Gatza et al. identified proliferation driving genes in non-basal breast cancer [3]. Amplification of four of these genes (MRPS23, FGD5, DTX3 and METTL6) was associated with a poor prognosis. Mitochondrial ribosomal protein S23 (MRPS23) is located on the long arm of chromosome 17 (17q22) and belongs to the mitochondrial ribosomal protein gene family $[4,5]$. Mitochondrial ribosomes are composed of a small $28 \mathrm{~S}$ subunit and a large $39 \mathrm{~S}$ subunit. MRPS23 encodes the $28 \mathrm{~S}$ subunit $[4,5]$. High MRPS23 expression has been found in colon [6], cervical $[7,8]$ and hepatocellular cancer $[9,10]$, and associated with poor prognosis in non-basal breast cancer [3], hepatocellular [10] and cervical cancer [7, 8]. In a breast cancer mouse model, MRPS23 knock-down reduced proliferation, induced apoptosis and limited angiogenesis and lymph node metastasis [11].

Our group has previously reclassified breast cancer tumours from a large cohort of Norwegian women into six molecular subtypes based on immunohistochemistry (IHC) and chromogenic in situ hybridization (CISH) [12]. The aims of the present study were to characterize MRPS23 copy number alterations by fluorescence in situ hybridization (FISH) on formalin-fixed, paraffin-embedded (FFPE) primary tumour tissue and corresponding lymph node metastases from this cohort, and to assess how these copy number alterations associates with molecular subtypes, proliferation and prognosis. Furthermore, using the METABRIC dataset [13], we assess how MRPS23 gene expression levels correlate with molecular subtypes and prognosis.

\section{Materials and methods}

\section{Study populations and specimen characteristics}

\section{Cohort 1}

Between 1956 and 1959, 25,727 women born 1886-1928 were invited to attend a clinical examination for early detection of breast cancer in Nord-Trøndelag County, Norway [14]. Through linkage with data from the Cancer Registry of Norway, these women were followed for breast cancer occurrence. Between 1961 and 2008, 1393 new breast cancers were registered. All tumours were reclassified according to histological type and grade [12, 15]. Tissue microarray (TMA) blocks were made using the Tissue Arrayer Mini-Core with TMA Designer2 software (Alphelys). Three 1-mm-in-diameter tissue cores from the periphery of the FFPE primary tumours and lymph node metastases were transferred to TMA recipient blocks. TMA sections $(4 \mu \mathrm{m})$ were cut and stained, and the tumours were reclassified into molecular subtypes. Human epidermal growth factor receptor 2 (HER2) was assessed using both CISH and IHC. Tumours with HER2/ chromosome enumeration probe 17 (CEP17) ratio $\geq 2$ were defined as HER2 positive. When CISH was unsuccessful, tumours with intense membranous staining (IHC 3+) in $>10 \%$ of tumour cells were considered HER 2 positive.

Of the 1393 tumours, 909 were successfully reclassified into molecular subtypes [12]:Luminal A (ER and/or PR+, HER2-, Ki67 < 15\%), Luminal B (HER2-) (ER+ and/ or PR,+ HER $2-$, Ki67 $\geq 15 \%)$, Luminal B (HER2+) $(\mathrm{ER}+$ and/or PR,+ HER2+), HER2 type (ER- and PR-, HER2+), 5 negative phenotype (5NP; ER-, PR-, HER2-, CK5 - and EGFR-) and Basal phenotype (BP; ER-, PR-, HER2 - , CK5+ and/or EGFR+) (Supplementary Fig. 1). From the time of diagnosis (baseline), patients were followed until death from breast cancer, death from other causes or until December 31st, 2015. Individual information about adjuvant treatment is unavailable. However, due to age and/or time of diagnosis, few would have received chemotherapy. Some would have been treated with antihormonal treatment, but none qualified for trastuzumab. In the present study, TMAs containing cores from tumours diagnosed mainly in the 1980 s or later $(n=636)$ were included. Of these, 46 were excluded due to unsuccessful FISH $(n=30)$ or insufficient amounts of tumour tissue $(n=16)$. Thus, 590 cases were suitable for MRPS23 and CEP17 copy number assessment. Of these, 192 had lymph node metastases, and lymph node tissue from 150 was available in TMAs. Due to unsuccessful FISH $(n=5)$ or insufficient amounts of tissue $(n=1)$, six were excluded. Hence, lymph node metastases from 144 cases were included.

\section{Cohort 2 METABRIC}

The METABRIC dataset includes a discovery dataset $(n=997)$, and a validation dataset $(n=995)$. The cohorts have previously been described in detail [13]. In the present study, 1971 cases had available follow-up data and MRPS23 gene expression data from all primary breast tumours. Tumours of basal-like $(n=329)$, normal-like $(n=202)$ and unknown subtype $(n=6)$ were excluded from our analysis $[3,16,17]$. Thus, 1434 tumours were included. Patients with ER positive and/or lymph node negative tumours had not received chemotherapy, whereas patients with ER negative and lymph node positive 
tumours did [13]. None of the HER2+ patients were treated with trastuzumab. To assess possible associations between gene expression levels and prognosis, cases were separated into quartiles. Prognosis for each quartile was analysed separately, and for dichotomization of gene expression values, patients with gene expression levels in the upper quartile were compared to all other cases.

\section{MRPS23 FISH cohort 1}

FISH was done according to the manufacturer's guidelines using Dako Histology FISH Accessory Kit K 579911. After de-waxing and rehydration, TMA slides were boiled in a microwave oven $(10 \mathrm{~min})$ in Pre-Treatment Solution, cooled (15 min) and washed in Wash Buffer $(2 \times 3 \mathrm{~min})$. Protein digestion was performed with Pepsin Solution at $37{ }^{\circ} \mathrm{C}(30 \mathrm{~min})$, and then washed in Wash buffer $(2 \times 3 \mathrm{~min})$. Dehydration was done in ethanol ( 70 , 80 and $95 \%$ ) for 2 min at each concentration, and the slides were then air dried at room temperature for $15 \mathrm{~min}$. FISH-custom probes for MRPS23 ( $3 \mu \mathrm{L}$, Empire Genomics) and CEP17 ( $1 \mu \mathrm{L}$, Abbott/VYSIS) were mixed with hybridization buffer ( $9 \mu \mathrm{L}$, Empire Genomics) and applied to TMA slides. Coverslips were applied and sealed with coverslip sealant (Dako). Denaturation was performed at $83{ }^{\circ} \mathrm{C}$ (3 min) followed by hybridization at $37{ }^{\circ} \mathrm{C}$ overnight in a DAKO Hybridizer. Post hybridization, TMA slides were rinsed in $0.4 \mathrm{xSSC} / 0.3 \% \mathrm{NP}-40$ at $72{ }^{\circ} \mathrm{C}(2 \mathrm{~min})$, and in $2 \mathrm{xSSC} / 0.1 \% \mathrm{NP}-40$ at RT $(15 \mathrm{~s})$. Slides were air dried at $37{ }^{\circ} \mathrm{C}(15 \mathrm{~min})$. DAPI (15 $\mu \mathrm{L}$, VYSIS. Abbott no 06J50001) was applied and the slides were coverslipped.

MRPS23 and CEP17 copy numbers were counted using a fluorescence microscope (Nikon Eclipse 90i). All available tissue cylinders from each case were examined, and MRPS23 and CEP17 copy number in 20 well-preserved, non-overlapping tumour cell nuclei were recorded. Mean copy number of MRPS23/tumour cell and MRPS23/CEP17 ratio were estimated for each case. To assess the impact of mean MRPS23 copy number, cases were divided into three categories based on recent HER2 guidelines [18]: mean MRPS23 copy number $<4$; mean $\geq 4<6$ and mean $\geq 6$. To estimate the impact of gene/centromere ratio, cases were divided into two categories: MRPS23/CEP17 <2 and MRPS23/ CEP17 $\geq 2$. Finally, MRPS23 amplification (MRPS23+) was defined as mean MRPS23 copy number $\geq 6$ and/or MRPS23/ CEP17 ratio $\geq 2$. Cases with $M R P S 23$ copy number $<6$ and $M R P S 23 / C E P 17$ ratio $<2$ were defined as non-amplified (MRPS23-). To study the prognostic value of HER2 status and MRPS23 status combined, cases were divided into four groups: MRPS23-/HER2-; MRPS23-/HER2+; MRPS23+/HER2 - and MRPS23+/HER2+. The REMARK criteria for tumour marker reporting were followed [19].

\section{Statistical analyses}

Pearson chi square tests were used to compare MRPS23 copy number status and MRPS23 gene expression levels in the primary tumours across patient and tumour characteristics. To compare copy number status in the primary tumours and their corresponding lymph node metastases, paired analyses were performed using McNemar's and marginal homogeneity test.

For each category of MRPS23 copy number status and $M R P S 23 / H E R 2$ status in primary tumours, and for the MRPS23 gene expression categories, we estimated cumulative incidence of death from breast cancer, with death from other causes as a competing event. We used Gray's test to compare equality of cumulative incidence curves. Cox proportional hazard models were used to estimate hazard ratios (HR) of breast cancer death with $95 \%$ confidence intervals (CI). In the Cox regression analyses, patients were censored at time of death from other causes. Where applicable, adjustments were made for age at baseline $(\leq 49,50-59$, 60-64, 65-69, 70-74, $\geq 75$ ), histological grade (I-III), stage (I-IV), Ki67 status $(</ \geq 15 \%)$ and molecular subtype. We found no clear violations of proportionality in log-minus-log plots. Linear regression analyses were used for comparison of MRPS23 expression levels between different molecular subtypes.

All statistical tests were two-sided, and statistical significance was assessed at 5\% level. $p$-values between 5 and $10 \%$ were regarded borderline significant. All statistical analyses were done using STATA version 15.1 (Stata Corp., College Station, TX, USA).

\section{Results}

\section{Cohort 1}

Mean age at diagnosis was 75.6 (SD 8.6, range 41-96) years and mean follow-up after diagnosis was 8.9 (SD 7.2) years. By the end of follow-up, 217 (37\%) patients had died from breast cancer, and 318 (54\%) had died from other causes (Table 1).

\section{MRPS23 copy number status in primary tumours}

When MRPS23 and CEP17 copy number alterations were present, a homogenous pattern was seen, with alterations in the majority of tumour cells. Three different phenotypes were seen, tumours without copy number alterations; tumours with MRPS23 and CEP17 copy number increase and tumours with MRPS23 copy number increase only (Fig. 1). In total, 29 of the primary tumours (5\%) had mean MRPS23 copy number $\geq 4<6,21$ (4\%) had 


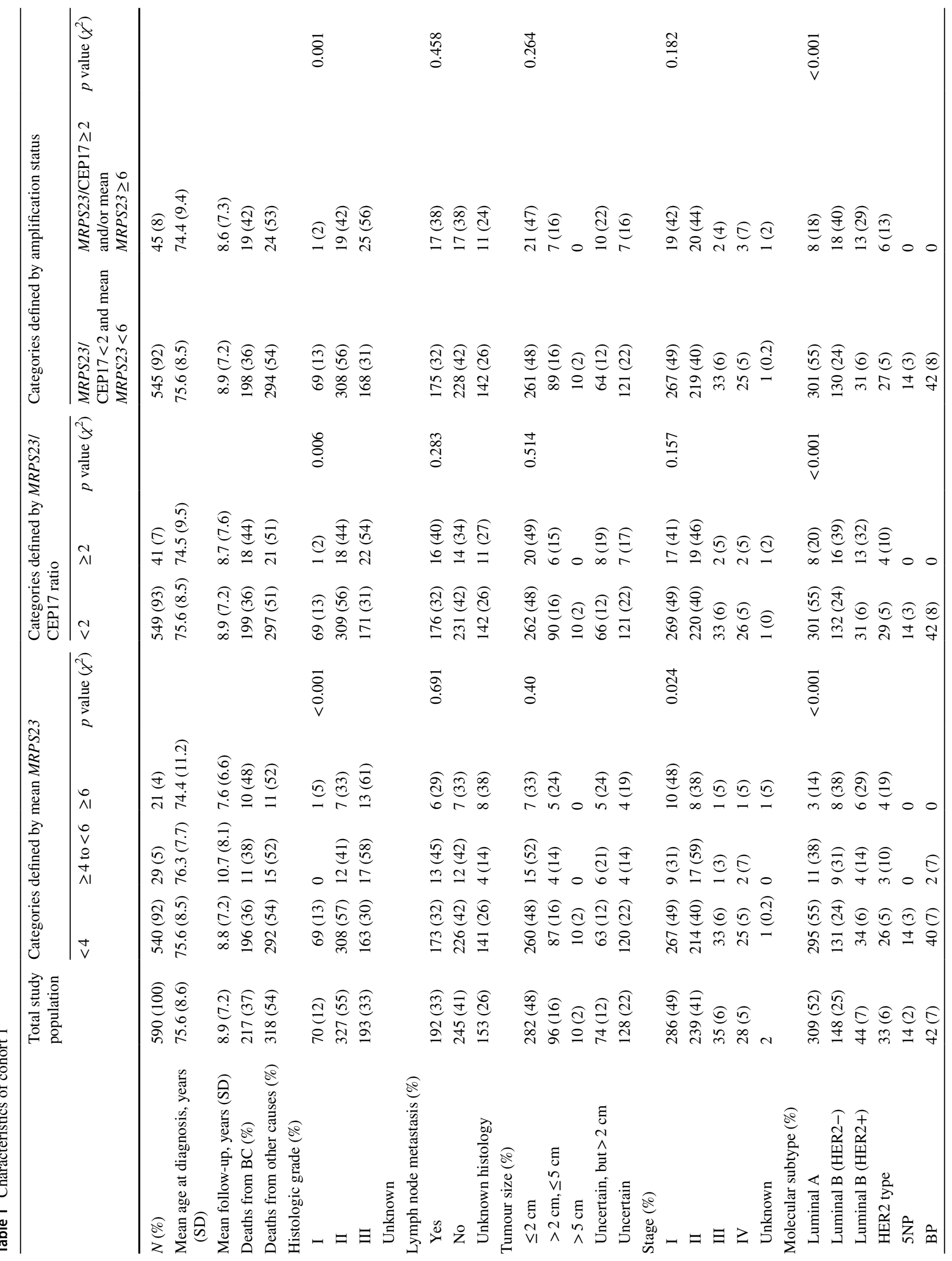




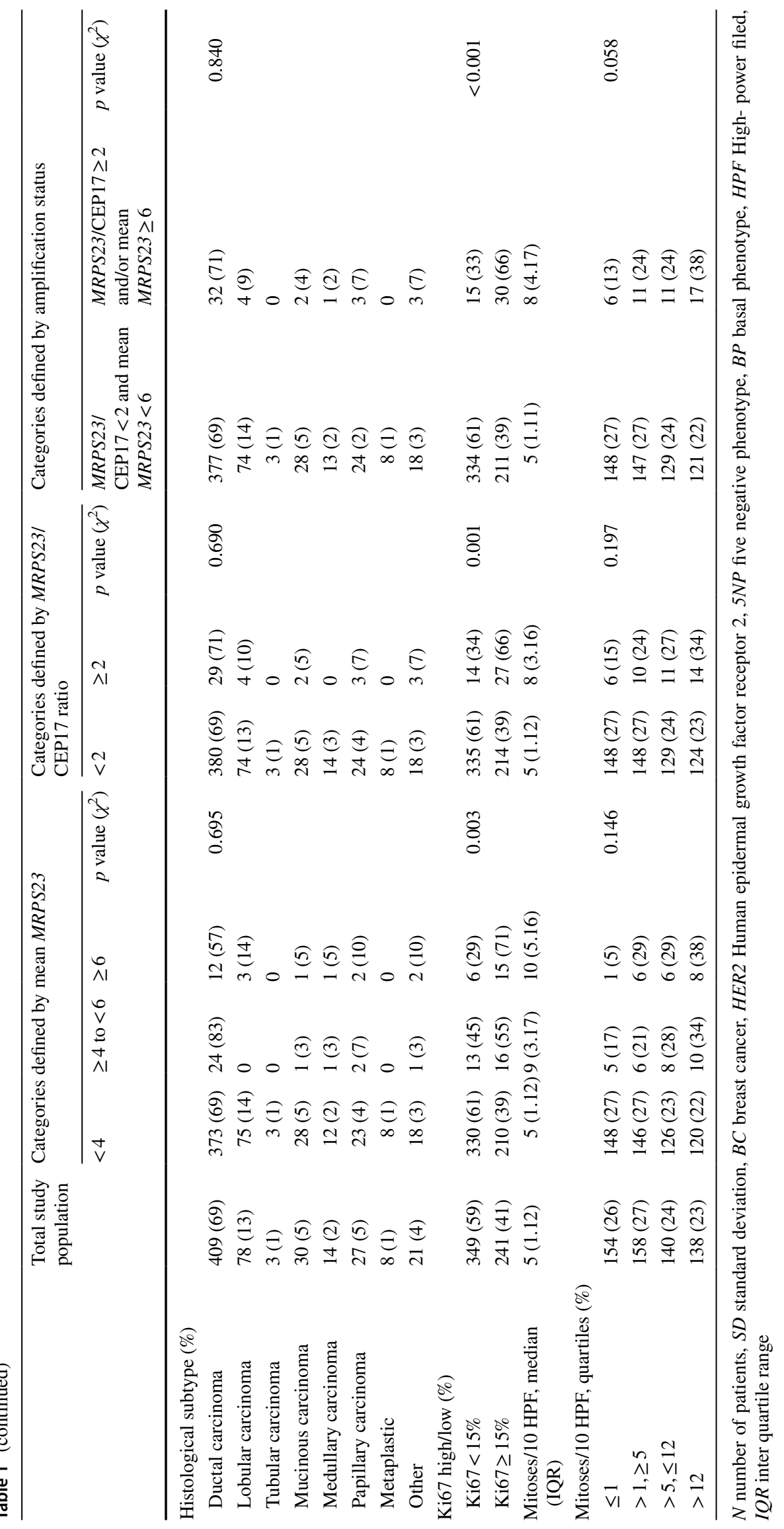



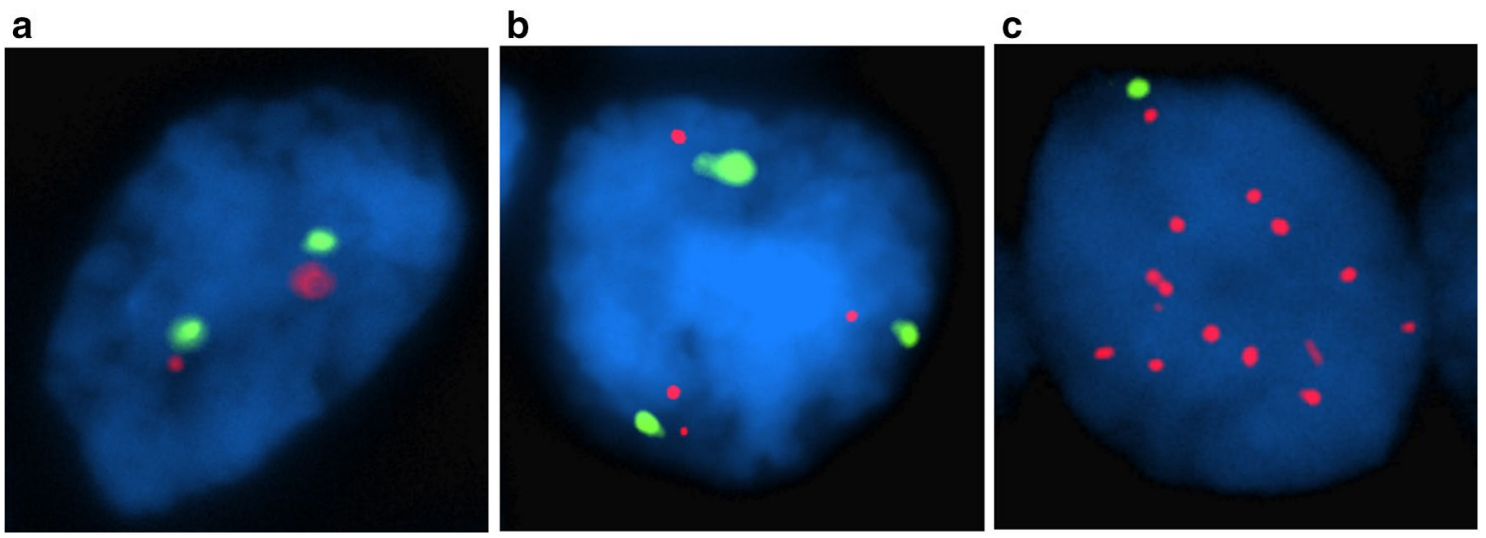

Fig. 1 Breast cancer cell nucleus with a two copies of MRPS23 and CEP17, b copy number increase of both MRPS23 and CEP17 and c copy number increase of MRPS23 without corresponding increase of CEP17

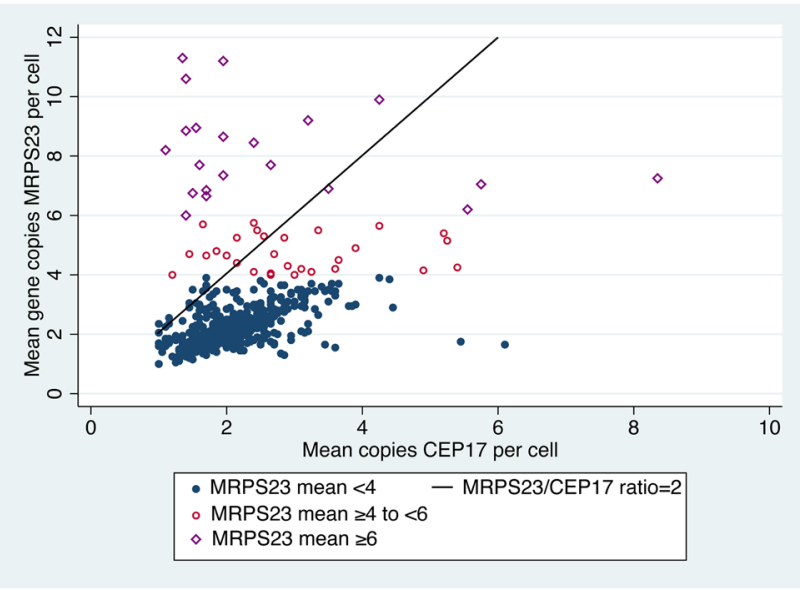

Fig. 2 Scatter plot of MRPS23 and CEP17 copy number in 590 breast cancer tumours

mean copy number $\geq 6$ and 41 (7\%) had MRPS23/CEP17 ratio $\geq 2$ (Table 1, Fig. 2). A total of 45 tumours ( $8 \%$ ) were amplified (mean MRPS23 $\geq 6$ and/or MRPS23/ CEP17 $\geq 2$ ). Among cases with mean $M R P S 23 \geq 6$, four cases had MRPS23/CEP17 ratio $<2$. Of cases with $M R P S 23 / C E P 17$ ratio $\geq 2,24$ had mean MRPS $23<6$.

MRPS23 copy number increase (mean $\geq 4$ ) was found within all molecular subtypes except the 5NP (Table 1). Amplifications were found in all molecular subtypes except the 5NP and the BP. Amplifications were seen in $30 \%$ of Luminal B (HER $2+$ ), $18 \%$ of HER 2 type, $12 \%$ of Luminal B (HER2-), and 3\% of Luminal A. Of the MRPS23 amplified tumours, 19 (42\%) were HER2+, compared to $58(11 \%)$ of non-amplified tumours.

\section{Copy number status in lymph node metastases}

In total, 144 cases were examined for MRPS23 copy number status in their lymph node metastases (Table 2). There were no significant changes in MRPS23 copy number status in the lymph node metastases compared to the corresponding primary tumours. Among the pairs of primary tumours and lymph node metastases, 14 (10\%) primary tumours were classified as MRPS23+, and in 10 of these (71\%), the corresponding lymph node metastases were also MRPS23+. $M R P S 23$ amplification was also identified in the lymph node metastases of two MRPS23-tumours.

\section{MRPS23 copy number status and proliferation}

Of the MRPS23 amplified tumours, 66\% had high Ki67 ( $\geq 15 \%$ ), compared to $39 \%$ of the non-amplified tumours (Table 1). Mitotic counts were also higher in amplified tumours (borderline significance). Of the MRPS23 amplified tumours, $56 \%$ were grade III, compared to $31 \%$ of the non-amplified tumours. An association between MRPS23 copy number increase and high Ki67, and high histological grade was also found when MRPS23 copy number status was defined by MRPS23 mean and MRPS23/CEP17 ratio (Table 1). In the lymph node metastases, MRPS23 amplification was associated with high Ki67 (borderline significance, Table 3).

\section{MRPS23 copy number status and prognosis}

\section{Mean MRPS23}

The cumulative risk of death from breast cancer for cases with no copy number increase was 35\% (95\% CI 31-39) 10 years after diagnosis (Table 4; Fig. 3a). The corresponding risks for cases with mean copy number $\geq 4<6$ 
Table 2 MRPS23 status in primary tumours and lymph node metastases according to MRPS23/CEP17 ratio, mean MRPS23 and amplification status

\begin{tabular}{lllll} 
Mean MRPS23/tumour cell, primary tumours & & & Marginal \\
\hline$<4$ & $\geq 4,<6$ & $\geq 6$ & Total & $\begin{array}{l}\text { homogeneity } \\
\text { test }\end{array}$
\end{tabular}

Mean MRPS23/tumour cell, lymph nodes

\begin{tabular}{llllrl}
$<4$ & $125(97)$ & $4(40)$ & $1(20)$ & 130 & $p=0.637$ \\
$\geq 4,<6$ & $3(2)$ & $5(50)$ & $2(40)$ & 10 & 4 \\
$\geq 6$ & $1(1)$ & $1(10)$ & $2(40)$ & 5 & 144 \\
Total & 129 & 10 & 5 & \\
\hline
\end{tabular}

MRPS23/CEP17 ratio, primary tumours

\begin{tabular}{llll}
\hline$<2$ & $\geq 2$ & Total & McNemar test
\end{tabular}

MRPS23/CEP17 ratio, lymph nodes

\begin{tabular}{lllr}
$<2$ & $130(99)$ & $3(23)$ & 133 \\
$\geq 2$ \\
$1(1)$ & $10(76)$ & 11 & $p=0.625$ \\
Total & 131 & 13 & 144 \\
\hline
\end{tabular}

Amplification status, primary tumours

\begin{tabular}{lllrr} 
& MRPS23- & MRPS23+ & Total & McNemar test \\
\cline { 4 - 5 } & & & 132 & $p=0.688$ \\
Amplification status, lymph nodes & $128(98)$ & $4(29)$ & 12 & 144 \\
MRPS23+ $^{\mathrm{b}}{ }^{\mathrm{b}}$ & $2(2)$ & $10(71)$ & 14 & 144 \\
Total & 130 & 14 & \\
\hline
\end{tabular}

${ }^{\mathrm{a}} M R P S 23 / \mathrm{CEP} 17<2$ and mean $M R P S 23<6$

${ }^{\mathrm{b}} \mathrm{MRPS} 23 / \mathrm{CEP} 17 \geq 2$ and/or mean $M R P S 23 \geq 6$

Table 3 MRPS23 amplification status and Ki67 levels in lymph nodes

\begin{tabular}{lllll}
\hline & \multicolumn{4}{l}{ MRPS23 amplification status, lymph node } \\
\cline { 2 - 5 } & MRPS23- $^{\mathrm{a}}$ & MRPS23+ & Total & $\chi^{2}$ \\
\hline Ki67, lymph node (\%) & & & & \\
Ki67<15\% & $69(53 \%)$ & $3(25 \%)$ & 72 & $p=0.07$ \\
Ki67 $>15 \%$ & $62(47 \%)$ & $9(75 \%)$ & 71 & \\
Total & 131 & 12 & 143 & \\
\hline
\end{tabular}

${ }^{\mathrm{a}} \mathrm{MRPS} 23 / \mathrm{CEP} 17<2$ and mean MRPS23 $<6$

${ }^{\mathrm{b}} M R P S 23 / \mathrm{CEP} 17 \geq 2$ and/or mean $M R P S 23 \geq 6$

and mean $\geq 6$ was $34 \%$ (95\% CI 20-55), and $43 \%$ (95\% CI 25-66), respectively. In the Cox regression analysis, there were no clear differences in the rates of death between categories. Adjustments for age, stage, grade, Ki67 or HER2 status did not influence the results.

\section{MRPS23/CEP17 ratio}

After 10 years of follow-up, cases with MRPS23/CEP17 ratio $<2$ had a cumulative risk of death from breast cancer of 30\% (95\% CI 27-34) (Table 4; Fig. 3b), whereas cases with ratio $\geq 2$ had a corresponding risk of $41 \%$ (95\% CI 30-58).
When comparing rates of death from breast cancer, there was no clear difference between the two categories (HR 1.2, 95\% CI 0.8-2.0). Adjusting for age, stage, grade, Ki67 or HER2 status did not influence the results.

\section{MRPS23 amplification status}

After 10 years of follow-up, patients with MRPS23 amplified tumours had $40 \%$ (95\% CI 27-56) cumulative risk of death from breast cancer, compared to $30 \%$ (95\% CI 27-34) for patients without amplification (Table 4; Fig. 3c). The rates of death from breast cancer were similar for cases with and without amplification (HR 1.2, 95\% CI 0.7-1.9). Separate adjustments for age, grade, histological grade, stage and HER2 status did not influence the results. Analysis of prognosis was also done for Luminal A cases separately, and for all luminal subtypes combined. In these analyses, no clear differences in prognosis between MRPS23 amplified and non-amplified cases were seen (data not shown).

\section{MRPS23/HER2- status}

When tumours were reclassified into four categories based on MRPS23 and HER2 status, the highest risk of death was 
Table 4 Absolute and relative risk of death from breast cancer according to mean MRPS23/tumour cell, MRPS23/CEP17 ratio and amplification status

\begin{tabular}{|c|c|c|c|c|c|c|c|}
\hline & \multicolumn{3}{|c|}{$\begin{array}{l}\text { Mean MRPS23/tumour cell, primary } \\
\text { tumour }\end{array}$} & \multicolumn{2}{|c|}{$\begin{array}{l}\text { MRPS23/CEP17 ratio, } \\
\text { primary tumour }\end{array}$} & \multicolumn{2}{|c|}{$\begin{array}{l}\text { Amplification status, pri- } \\
\text { mary tumours }\end{array}$} \\
\hline & $<4$ & $\geq 4,<6$ & $\geq 6$ & $<2$ & $\geq 2$ & $\operatorname{MRPS23}-^{\mathrm{a}}$ & $\operatorname{MRPS} 23+^{\mathrm{b}}$ \\
\hline Cum. risk after 5 years $(\%)(95 \%$ CI) & $21(18-24)$ & $24(12-44)$ & $38(21-62)$ & $21(18-25)$ & $29(18-46)$ & $21(18-25)$ & $29(18-44)$ \\
\hline Cum. risk after 10 years (\%) (95\% CI) & $35(31-39)$ & $34(20-55)$ & $43(25-66)$ & $30(27-34)$ & $41(30-58)$ & $30(27-34)$ & $40(27-56)$ \\
\hline HR, unadjusted $(95 \% \mathrm{CI})$ & 1.0 & $0.9(0.5-1.7)$ & $1.5(0.8-2.8)$ & 1.0 & $1.2(0.8-2.0)$ & 1.0 & $1.2(0.7-1.9)$ \\
\hline HR adjusted for age ( $95 \% \mathrm{CI})$ & 1.0 & $0.9(0.5-1.7)$ & $1.5(0.8-2.8)$ & 1.0 & $1.3(0.8-2.1)$ & 1.0 & $1.2(0.7-1.9)$ \\
\hline HR adjusted for stage $(95 \% \mathrm{CI})$ & 1.0 & $0.8(0.4-1.5)$ & $1.2(0.6-2.4)$ & 1.0 & $1.2(0.7-1.9)$ & 1.0 & $1.0(0.6-1.7)$ \\
\hline HR adjusted for grade $(95 \% \mathrm{CI})$ & 1.0 & $0.7(0.4-1.4)$ & $1.3(0.7-2.4)$ & 1.0 & $1.1(0.7-1.7)$ & 1.0 & $1.0(0.6-1.6)$ \\
\hline HR adjusted for Ki67 (95\% CI) & 1.0 & $0.7(0.4-1.4)$ & $1.1(0.6-2.2)$ & 1.0 & $1.0(0.6-1.6)$ & 1.0 & $1.0(0.6-1.6)$ \\
\hline HR adjusted for HER2 $(95 \% \mathrm{CI})$ & 1.0 & $0.9(0.5-1.6)$ & $1.2(0.6-2.3)$ & 1.0 & $1.0(0.6-1.7)$ & 1.0 & $1.0(0.6-1.6)$ \\
\hline
\end{tabular}

CI confidence interval, $H R$ hazard ratio

${ }^{\mathrm{a}} M R P S 23 / \mathrm{CEP} 17<2$ and mean MRPS23 $<6$

${ }^{\mathrm{b}} M R P S 23 / \mathrm{CEP} 17 \geq 2$ and/or mean MRPS23 $\geq 6$

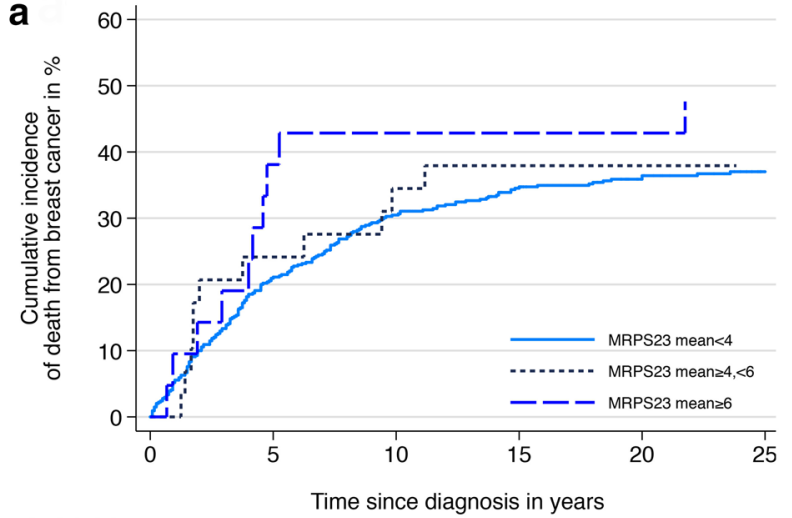

$$
\text { At risk (n) }
$$
MRPS23 mean<4 540 MRPS23 mean $\geq 4<6$, 540 $\begin{array}{ll}\text { MRPS23 mean } \geq 6 & 21\end{array}$

$\begin{array}{ccccc}427 & 357 & 310 & 240 & 16 \varsigma \\ 22 & 19 & 10 & 6 & 1 \\ 13 & 12 & 12 & 12 & 0\end{array}$

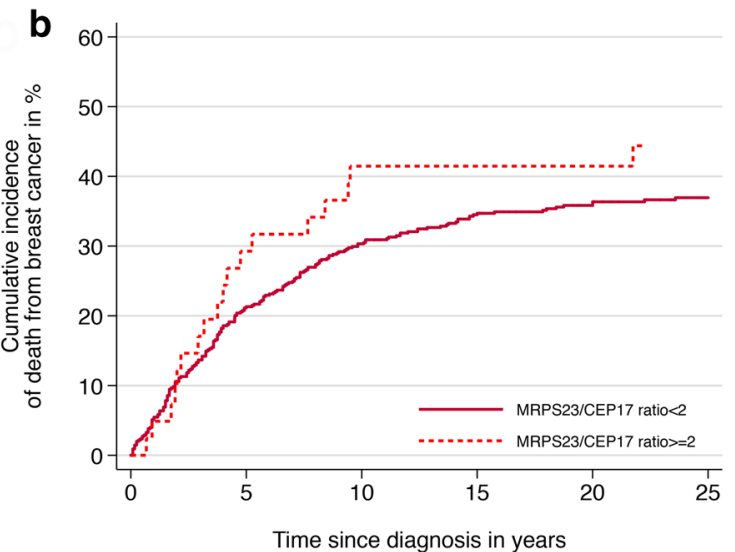

At risk (n)

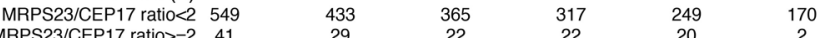

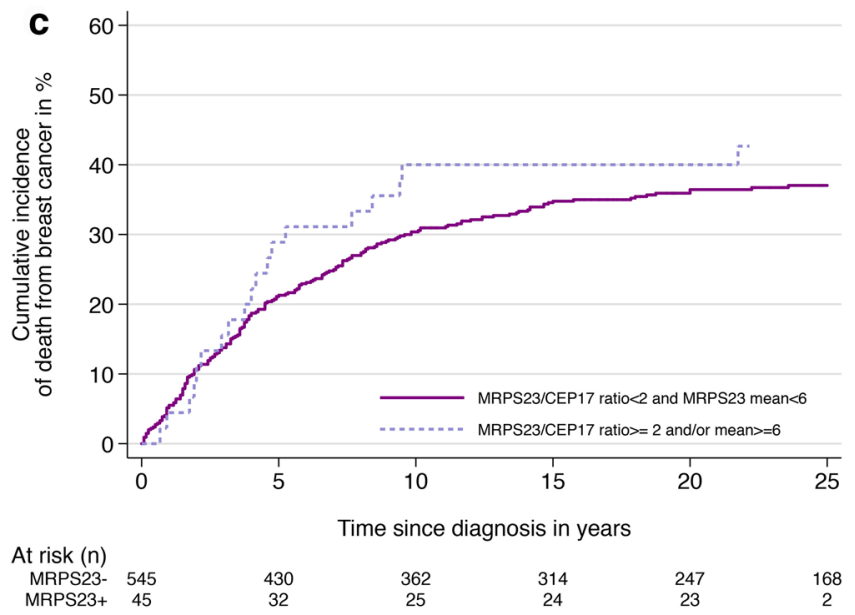

Fig. 3 Cumulative incidence of death from breast cancer according to MRPS23 copy number status based on a mean MRPS23 copy number $(p=0.47), \mathbf{b}$ MRPS23/CEP17 ratio $(p=0.29)$ and $\mathbf{c} M R P S 23$ amplification status $(p=0.39)$ 
Table 5 Absolute and relative risk of dying from breast cancer according to HER2 status and amplification of MRPS23

\begin{tabular}{lllll}
\hline & MRPS23- ${ }^{\mathrm{a}}$ HER2- & MRPS23-/HER2+ & MRPS23+ ${ }^{\mathrm{b}} / \mathrm{HER} 2-$ & MRPS23+/HER2+ \\
\hline Cum. risk after 5 years (\%) (95\% CI) & $18(15-22)$ & $44(32-58)$ & $12(4-32)$ & $53(33-76)$ \\
Cum. risk after 10 years (\%) (95\% CI) & $28(25-32)$ & $47(36-61)$ & $23(11-44)$ & $63(42-83)$ \\
HR, unadjusted (95\% CI) & 1.0 & $1.6(1.1-2.4)$ & $0.6(0.3-1.4)$ & $2.6(1.5-4.5)$ \\
HR adjusted for age (95\% CI) & 1.0 & $1.7(1.1-2.5)$ & $0.6(0.3-1.4)$ & $2.6(1.5-4.8)$ \\
HR adjusted for grade (95\% CI) & 1.0 & $1.2(0.8-1.8)$ & $0.5(0.2-1.1)$ & $2.3(1.3-4.0)$ \\
HR adjusted for stage (95\% CI) & 1.0 & $1.9(1.3-2.8)$ & $0.6(0.3-1.4)$ & $1.8(1.0-3.3)$ \\
HR adjusted for Ki67 (95\% CI) & 1.0 & $1.3(0.9-1.9)$ & $0.5(0.2-1.0)$ & $2.2(1.3-3.0)$ \\
HR adjusted for HER2 (95\% CI) & 1.0 & $1.6(1.1-2.4)$ & $0.6(0.3-1.4)$ & $2.6(1.5-4.5)$ \\
\hline
\end{tabular}

Cum. risk cumulative risk, $C I$ confidence interval, $H R$ hazard ratio

${ }^{\mathrm{a}} M P R 23-:$ MPRS23/CEP17 ratio $<2$ and MRPS23 mean $<6$

${ }^{\mathrm{b}}$ MRPS23 + : MRPS23/CEP17 ratio $\geq 2$ and/or MRPS23 mean $\geq 6$

${ }^{\mathrm{c}} H E R 2 / \mathrm{CEP} 17$ ratio $\geq 2$ or intense membranous staining (IHC $3+$ ) in $>10 \%$ of tumour cells

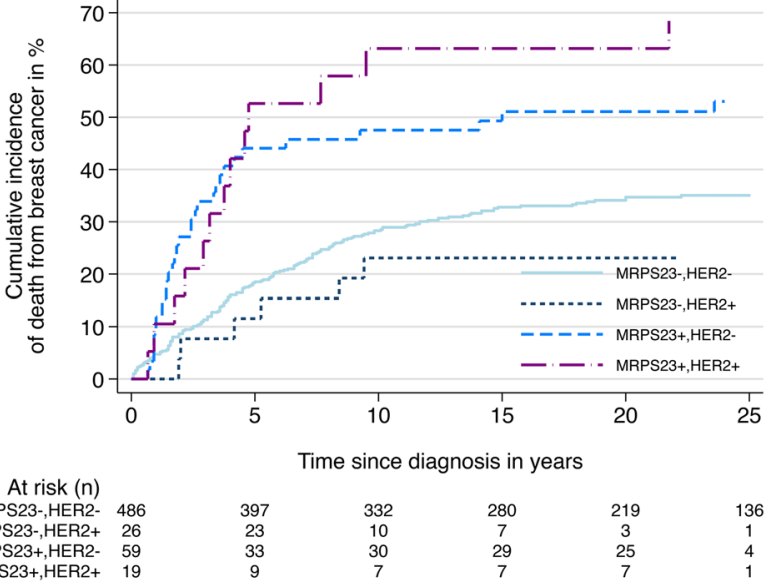

Fig. 4 Cumulative incidence of death from breast cancer according to MRPS23 amplification status and HER2 status $(p<0.01)$

found in the MRPS23+/HER2+subtype (Table 5; Fig. 4). After 10 years of follow-up, patients with the MRPS23+l HER2 + subtype had a cumulative risk of death from breast cancer of $63 \%$ (95\% CI 42-83). The corresponding risk for the MRPS23-/HER2+ subtype was 47\% (95\% CI 36-61). The lowest risk of death was found in the MRPS23+/HER2subtype (23\%, 95\% CI 11-44). There were no clear differences in the rate of death from breast cancer between the MRPS23-/HER2+ and MRPS23+/HER2+ subtypes (HR $1.3,95 \%$ CI 0.7-2.6). Separate analysis of prognosis was also done for all luminal cases, with similar results as for all cases combined (data not shown).

\section{Cohort 2}

\section{MRPS23 expression according to molecular subtypes}

In the METABRIC dataset, mean age at diagnosis was 61.1 (SD 12.4, range 22-96) years, and mean follow-up after diagnosis was 8.1 (SD 4.9) years. Characteristics of the study population are given in Table 6 . By the end of followup, 506 (26\%) patients had died from breast cancer, and 384 (20\%) had died from other causes.

High MRPS23 expression levels were associated with the Luminal B subtype (Fig. 5).

After 10 years of follow-up, cases with low MRPS23 expression levels had a cumulative risk of death from breast cancer of $26 \%$ (95\% CI 23-29\%), compared to $24 \%$ (95\% CI 20-30\%) among cases with high MRPS23 expression (Cut-off upper quartile; Fig. 6, Table 7). Comparing the rates of death from breast cancer, there were no significant differences between cases with gene expression levels in the upper quartile compared to the rest (HR 0.9, 95\% CI 0.7-1.2, Fig. 6, Table 7). Similar results were obtained when analysis of prognosis was done for each quartile separately. Adjustments for age and histological grade did not influence the results.

\section{Discussion}

We identified MRPS23 amplification in $8 \%$ of primary tumours and $9 \%$ of lymph node metastases in a large population of Norwegian breast cancer patients. The highest proportion of amplified cases was found within Luminal B (HER2+), HER2 type and Luminal B (HER2-) tumours. None of the amplified tumours were triple negative (5NP/ BP). MRPS23 amplification was associated with high Ki67 and high histological grade. No clear association between 
Table 6 Characteristics of Cohort 2 (METABRIC) (Normal-like and basal-like subtypes excluded from the analyses)

\begin{tabular}{|c|c|c|c|c|c|c|}
\hline & \multicolumn{4}{|c|}{ Total study population } & \multirow[t]{3}{*}{ Discovery cohort } & \multirow[t]{3}{*}{ Validation cohort } \\
\hline & \multirow[t]{2}{*}{ Total } & \multicolumn{2}{|c|}{ Mean probe $M R P S 23$} & \multirow[t]{2}{*}{$\chi^{2}$} & & \\
\hline & & Quartile $1-3^{\mathrm{a}}$ & Quartile $4^{\mathrm{b}}$ & & & \\
\hline$N(\%)$ & 1434 & 1069 & 365 & & $804(56)$ & $630(44)$ \\
\hline Mean age at diagnosis, years (SD) & $63.2(12.4)$ & $63.6(12.4)$ & $62(12.4)$ & & $62.3(12.5)$ & $64.3(12.2)$ \\
\hline Mean follow-up, years (SD) & $8.3(4.9)$ & $8.5(5)$ & $7.8(4.5)$ & & $8.1(4.7)$ & $8.5(5.1)$ \\
\hline Deaths from BC $(\%)$ & $354(25)$ & $273(25)$ & $81(22)$ & & $201(25)$ & $153(24)$ \\
\hline Deaths from other causes (\%) & $311(22)$ & $240(22)$ & $71(19)$ & & $156(19)$ & $155(25)$ \\
\hline \multicolumn{7}{|l|}{ Histologic grade (\%) } \\
\hline I & $139(10)$ & $116(11)$ & $23(6)$ & $<0.001$ & $61(8)$ & $78(12)$ \\
\hline II & $635(44)$ & $504(47)$ & $131(36)$ & & $375(47)$ & $260(41)$ \\
\hline III & $598(42)$ & $392(17)$ & $206(56)$ & & $368(46)$ & $230(37)$ \\
\hline Unknown & $62(4)$ & $57(5)$ & $5(1)$ & & 0 & $62(10)$ \\
\hline \multicolumn{7}{|l|}{ Lymph node metastasis (\%) } \\
\hline Yes & $684(48)$ & $491(46)$ & $193(53)$ & 0.030 & $383(48)$ & $301(48)$ \\
\hline No & $750(52)$ & $578(54)$ & $172(47)$ & & $421(52)$ & $329(52)$ \\
\hline \multicolumn{7}{|l|}{ Tumour size (\%) } \\
\hline$\leq 2 \mathrm{~cm}$ & $613(43)$ & $472(44)$ & $141(39)$ & 0.170 & $351(42)$ & $262(42)$ \\
\hline$>2 \mathrm{~cm}, \leq 5 \mathrm{~cm}$ & $751(52)$ & $542(51)$ & $209(57)$ & & $421(52)$ & $330(53)$ \\
\hline$>5 \mathrm{~cm}$ & $69(5)$ & $54(5)$ & $15(4)$ & & $32(4)$ & $37(6)$ \\
\hline Uncertain & $1(0)$ & $1(0)$ & 0 & & 0 & $1(0)$ \\
\hline \multicolumn{7}{|l|}{ Stage $(\%)$} \\
\hline I & $104(7)$ & $84(8)$ & $20(5)$ & 0.075 & 0 & $104(17)$ \\
\hline II & $177(12)$ & $141(13)$ & $36(10)$ & & 0 & $177(28)$ \\
\hline III & $27(2)$ & $20(2)$ & $7(2)$ & & 0 & $27(4)$ \\
\hline IV & $1(0)$ & 0 & $1(0)$ & & 0 & $1(0)$ \\
\hline Unknown & $1125(78)$ & $824(77)$ & $301(82)$ & & $804(100)$ & $321(50)$ \\
\hline \multicolumn{7}{|l|}{ PAM50 subtype (\%) } \\
\hline Luminal A & 709 (49) & $606(57)$ & $103(28)$ & & $445(56)$ & $255(40)$ \\
\hline Luminal B & $488(34)$ & $276(26)$ & $212(58)$ & & $266(33)$ & $222(35)$ \\
\hline HER2-type & 237 (17) & 187 (17) & $50(14)$ & $<0.001$ & $84(10)$ & $153(25)$ \\
\hline
\end{tabular}

$N$ number of patients, $S D$ standard deviation, $B C$ breast cancer

${ }^{a}$ Mean probe $M R P S 23 \leq 8.31$

${ }^{\mathrm{b}}$ Mean probe $M R P S 23>8.31$

MRPS23 amplification and prognosis was seen. The proportion of HER2 positive cases was higher among MRPS23 amplified cases, compared to non-amplified. MRPS23+/ HER2+ had the poorest prognosis.

In the METABRIC dataset, Luminal B tumours had the highest level of MRPS23 gene expression. We found no statistically significant associations between MRPS23 expression levels and prognosis.

This study is based on a well-described cohort of breast cancer patients with long-term follow-up, and data from the METABRIC dataset. In the Norwegian cohort, the majority of patients have been followed until death [12]. Since relapse may occur even decades after the primary diagnosis, long-term follow-up is of particular value in breast cancer research. Molecular subtyping was performed in the same laboratory, using the same algorithm and antibodies in all cases [12]. Using FISH, gene copy number can be assessed while observing the morphology of the tumour, ensuring that only invasive tumour cells were examined.

MRPS23 copy number in primary tumours and lymph node metastases was assessed in TMAs. In the primary tumours, tissue for TMAs was taken from the tumour periphery. Previous studies have shown good correlation between TMAs and corresponding whole sections [20,21]. Nevertheless, TMAs represent a small portion of each tumour, and, while copy number changes were observed throughout the tissue in amplified cases, intra tumour heterogeneity may not 


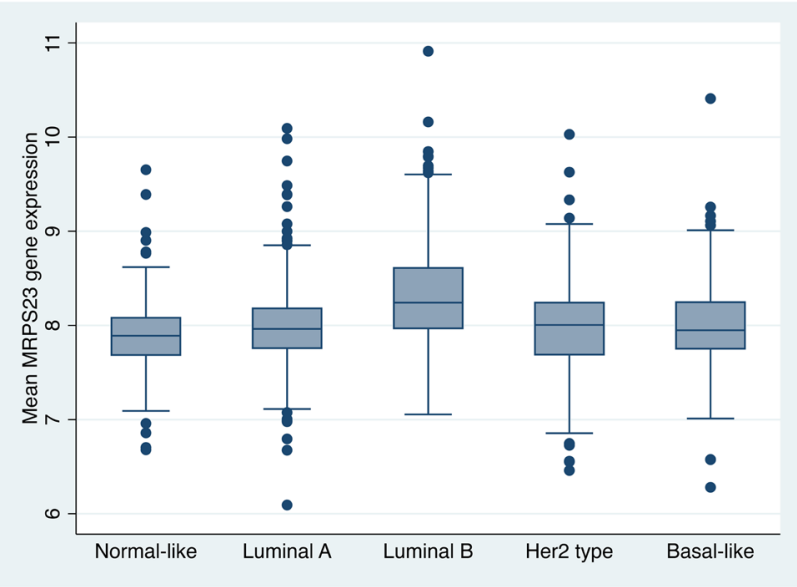

Fig. 5 MRPS23 gene expression according to molecular subtype in 1971 patients from the METABRIC dataset

be captured. It would therefore be of interest to validate our findings in a study of whole sections.

There are no established guidelines as to how MRPS23 amplification should be defined. According to HER2 ISH guidelines, both HER2 copy number and HER2/CEP17-ratio are taken into consideration [18]. MRPS23 and HER2 are both located on the long arm of chromosome 17 [4, 5, 22], and we chose to define MRPS23 amplification according to HER2 ISH guidelines, including both mean MRPS23 copy number and MRPS23/CEP17 ratio in our definition. Previous studies have shown a high frequency of abnormalities on chromosome 17, but rarely true polysomy [23]. The number of amplified cases was increased when including MRPS23/ CEP17 ratio in addition to mean MRPS23 in the definition of amplification. Hence, our definition may have led to overestimation of MRPS23 amplified cases. Nevertheless, we found that $8 \%$ of tumours were MRPS23 amplified, whereas $20 \%$ and $33 \%$ of the tumours were amplified in the two datasets included in Gatza et al. In the latter two cohorts, only luminal (defined as non-basal) cases were included. When excluding the BP and 5NP in our in-house cohort, the proportion of amplified cases was still 8\%. In accordance with other studies we found that amplification of MRPS23 was associated with higher proliferation $[3,11]$. However, contrary to others, we found no clear associations between MRPS23 copy number increase and a poorer prognosis [3]. Our study demonstrates the importance of validating biomarkers identified by high-throughput genomic analyses. Validation analyses of single biomarkers with FISH, performed in FFPE tissue, indicate the marker's prognostic potential when assessed in a routine diagnostic setting.

MRPS23 has previously been found to be amplified exclusively in highly proliferative luminal tumours [3]. In that study, PAM50 was used for molecular subtyping, and "luminal" was defined as all tumours that were not basal [3]. This definition of luminal was based on a study showing that breast tumours could be separated into two main groups, one group containing luminal and HER2-positive tumours and the other group comprising basal-like tumours [17]. In our study, cohort 1 was divided into six subtypes based on IHC and ISH. Although it has been shown that surrogate markers can be used for molecular subtyping [24-27], there is a discrepancy between molecular subtype defined by surrogate markers and subtypes defined by gene expression analyses $[28,29]$. Nevertheless, similar to Gatza et al., we only found MRPS23 amplified cases among the non-basal tumours.

Contrary to others [3], we found no clear associations between MRPS23 amplification and prognosis in our cohort of Norwegian breast cancer patients. We used a different method for assessment of gene copy number in
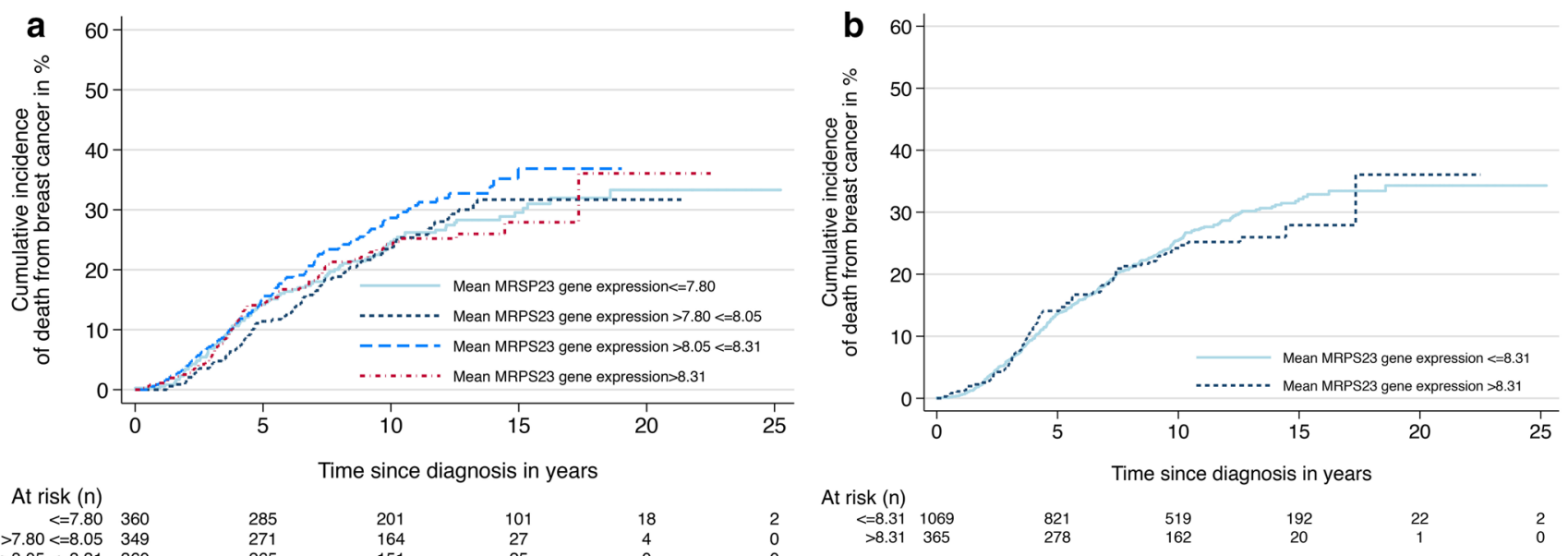

\begin{tabular}{|c|c|c|c|c|c|c|}
\hline \multicolumn{7}{|l|}{ At risk (n) } \\
\hline$<=7.80$ & 360 & 285 & 201 & 101 & 18 & 2 \\
\hline$>7.80<=8.0$ & 349 & 271 & 164 & 27 & 4 & 0 \\
\hline$>8.05<=8.3$ & 360 & 265 & 151 & 25 & 0 & 0 \\
\hline$>8.31$ & 365 & 278 & 162 & 20 & 1 & 0 \\
\hline
\end{tabular}

Fig. 6 Cumulative incidence of death from breast cancer according to MRPS23 gene expression divided into a quartiles 1-4 ( $p=0.4$ ), and $\mathbf{b}$ quartile $1-3$ vs. quartile $4(p=0.6)$ 
Table 7 Absolute and relative risk of death from breast cancer according to gene expression levels of MRPS23, METABRIC data

\begin{tabular}{|c|c|c|c|c|c|c|}
\hline & \multicolumn{4}{|c|}{ Mean probe MRPS23, quartiles } & \multicolumn{2}{|c|}{ Mean probe MRPS23 } \\
\hline & Quartile $1^{\mathrm{a}}$ & Quartile $2^{\mathrm{b}}$ & Quartile $3^{c}$ & Quartile $4^{\mathrm{d}}$ & Quartile 1-3 & Quartile 4 \\
\hline $\begin{array}{l}\text { Cum. risk after } \\
5 \text { years }(\%) \\
(95 \% \mathrm{CI})\end{array}$ & $14(11-18)$ & $11(8-15)$ & $15(12-20)$ & $14(11-18)$ & $14(12-16)$ & $14(11-18)$ \\
\hline $\begin{array}{l}\text { Cum. risk after } \\
10 \text { years }(\%) \\
(95 \% \mathrm{CI})\end{array}$ & $25(20-30)$ & $23(19-29)$ & $29(24-34)$ & $24(20-30)$ & $26(23-29)$ & $24(20-30)$ \\
\hline $\begin{array}{l}\text { HR, unadjusted } \\
\quad(95 \% \mathrm{CI})\end{array}$ & 1 & $1.0(0.7-1.3)$ & $1.2(0.9-1.6)$ & $1.0(0.7-1.3)$ & 1 & $0.9(0.7-1.2)$ \\
\hline $\begin{array}{l}\text { HR, adjusted for } \\
\text { age }(95 \% \mathrm{CI})\end{array}$ & 1 & $1.0(0.7-1.4)$ & $1.2(0.9-1.6)$ & $1.0(0.7-1.4)$ & 1 & $0.9(0.7-1.2)$ \\
\hline $\begin{array}{l}\text { HR, adjusted for } \\
\text { grade }(95 \% \mathrm{CI})\end{array}$ & 1 & $1.0(0.7-1.4)$ & $1.2(0.9-1.6)$ & $0.9(0.7-1.2)$ & 1 & $0.8(0.7-1.1)$ \\
\hline
\end{tabular}

Cum. risk cumulative risk, $C I$ confidence interval, $H R$ hazard ratio; Luminal A, Luminal B and HER2-type included in analyses

${ }^{\mathrm{a}}$ Mean probe $\operatorname{MRPS} 23 \leq 7.80$

${ }^{\mathrm{b}}$ Mean probe $M R P S 23>7.80, \leq 8.05$

${ }^{\mathrm{c}}$ Mean probe $M R P S 23>8.05, \leq 8.31$

${ }^{\mathrm{d}}$ Mean probe $\operatorname{MRPS} 23>8.31$

our study (FISH), and different methodologies could partly explain the divergent results. Furthermore, the number of amplified cases was low in our study population, and the results must be interpreted with caution. Patient age in Cohort 1 is high, and the majority of patients were not given modern breast cancer treatment either due to their age at diagnosis or time of diagnosis [12]. This enables us to follow the near-natural course of disease after surgery. It would, however, be interesting to perform a MRPS23 FISH study in a cohort of younger patients treated according to current guidelines.

Interestingly, the MRPS23+/HER2+ tumours had the poorest prognosis. HER 2 is recognized as an important prognostic marker in breast cancer, and interaction with MRPS23 could potentially be of clinical importance. However, due to the low number of cases in some of the MRPS23/HER2 categories, the results should be interpreted with caution.

We found no correlation between MRPS23 expression levels and prognosis in the METABRIC data set. In our analyses of prognosis, we excluded basal-like and normal-like cases [3,16]. A correlation between MRPS23 copy number status and gene expression has previously been found in the METABRIC dataset [3]. Since transcription is regulated by several mechanisms, good correlation between gene copy number and gene expression is infrequent [30-32]. Our analyses show that MRPS23 expression levels were overlapping between the different molecular subtypes. Such overlap was also seen between the highly proliferative luminal tumours (Luminal B) and basal-like tumours, the latter shown to be non-amplified. This could possibly be due to other MRPS23 up-regulating mechanisms, and potentially explain the lack of correlation between gene expression levels and prognosis in the METABRIC dataset.

\section{Conclusion}

Using FISH on a large cohort of breast cancer patients we found that MRPS23 amplification is associated with higher tumour cell proliferation. Amplifications were only found in luminal and HER2 type tumours. We found no association between MRPS23 amplification and prognosis. In the METABRIC dataset, gene expression levels were highest in Luminal B tumours. There was no correlation between MRPS23 expression and prognosis.

Acknowledgements Open Access funding provided by NTNU Norwegian University of Science and Technology (incl St. Olavs Hospital - Trondheim University Hospital). The study received financial support from the Medical Student Research Program at NTNU and UNIFOR. The authors thank the Department of Pathology, St. Olav's Hospital, Trondheim University Hospital for making the archives available for the study and the Cancer Registry of Norway for providing the patient data.

\section{Compliance with ethical standards}

Conflict of interest All authors declare that they have no conflicts of interest.

Ethical approval All procedures performed in studies involving human participants were in accordance with the ethical standards of the institutional and/or national research committee and with the 1964 Helsinki Declaration and its later amendments or comparable ethical standards. This study was approved, including dispensation from the requirement 
of patient consent, by the Regional committee for medical research ethics (REK 836-09).

Open Access This article is licensed under a Creative Commons Attribution 4.0 International License, which permits use, sharing, adaptation, distribution and reproduction in any medium or format, as long as you give appropriate credit to the original author(s) and the source, provide a link to the Creative Commons licence, and indicate if changes were made. The images or other third party material in this article are included in the article's Creative Commons licence, unless indicated otherwise in a credit line to the material. If material is not included in the article's Creative Commons licence and your intended use is not permitted by statutory regulation or exceeds the permitted use, you will need to obtain permission directly from the copyright holder. To view a copy of this licence, visit http://creativecommons.org/licenses/by/4.0/.

\section{References}

1. Hanahan D, Weinberg RA (2000) The hallmarks of cancer. Cell 100(1):57-70

2. Hanahan D, Weinberg RA (2011) Hallmarks of cancer: the next generation. Cell 144(5):646-674. https://doi.org/10.1016/j. cell.2011.02.013

3. Gatza ML, Silva GO, Parker JS, Fan C, Perou CM (2014) An integrated genomics approach identifies drivers of proliferation in luminal-subtype human breast cancer. Nat Genet 46(10):10511059. https://doi.org/10.1038/ng.3073

4. MRPS23 mitochondrial ribosomal protein S23 Homo sapiens (human) (2019) NCBI (National Center for Biotechnology Information). https://www.ncbi.nlm.nih.gov/gene/51649. Accessed 9 Jan 2019

5. Kenmochi N, Suzuki T, Uechi T, Magoori M, Kuniba M, Higa S, Watanabe K, Tanaka T (2001) The human mitochondrial ribosomal protein genes: mapping of 54 genes to the chromosomes and implications for human disorders. Genomics 77(1):65-70

6. Staub E, Gröne J, Mennerich D, Röpcke S, Klamann I, Hinzmann B, Castanos-Velez E, Mann B, Pilarsky C, Brümmendorf T, Weber B, Buhr HJ, Rosenthal A (2006) A genome-wide map of aberrantly expressed chromosomal islands in colorectal cancer. Mol Cancer 5:37. https://doi.org/10.1186/1476-4598-5-37

7. Li B, Zhang YL (2002) Identification of up-regulated genes in human uterine leiomyoma by suppression subtractive hybridization. Cell Res 12:215. https://doi.org/10.1038/sj.cr.7290127

8. Lyng H, Brøvig RS, Svendsrud DH, Holm R, Kaalhus O, Knutstad K, Oksefjell H, Sundfør K, Kristensen GB, Stokke T (2006) Gene expressions and copy numbers associated with metastatic phenotypes of uterine cervical cancer. BMC Genomics 7(1):268

9. Kurokawa Y, Matoba R, Nakamori S, Takemasa I, Nagano H, Dono K, Umeshita K, Sakon M, Monden M, Kato K (2004) PCRarray gene expression profiling of hepatocellular carcinoma. J Exp Clin Cancer Res 23(1):135-142

10. Pu M, Wang J, Huang Q, Zhao G, Xia C, Shang R, Zhang Z, Bian Z, Yang X, Tao K (2017) High MRPS23 expression contributes to hepatocellular carcinoma proliferation and indicates poor survival outcomes. Tumour Biol 39(7):1010428317709127. https:// doi.org/10.1177/1010428317709127

11. Gao Y, Li F, Zhou H, Yang Y, Wu R, Chen Y, Li W, Li Y, Xu $X$, Ke C, Pei Z (2017) Down-regulation of MRPS23 inhibits rat breast cancer proliferation and metastasis. Oncotarget 8(42):71772-71781. https://doi.org/10.18632/oncotarget.17888

12. Engstrom MJ, Opdahl S, Hagen AI, Romundstad PR, Akslen LA, Haugen OA, Vatten LJ, Bofin AM (2013) Molecular subtypes, histopathological grade and survival in a historic cohort of breast cancer patients. Breast Cancer Res Treat 140(3):463-473. https ://doi.org/10.1007/s10549-013-2647-2

13. Curtis C, Shah SP, Chin S-F, Turashvili G, Rueda OM, Dunning MJ, Speed D, Lynch AG, Samarajiwa S, Yuan Y (2012) The genomic and transcriptomic architecture of 2,000 breast tumours reveals novel subgroups. Nature 486(7403):346

14. Kvale GHI, Eide GE (1987) A prospective study of reproductive factors and breast cancer. Am J Epidemiol 126(5):831-841

15. Lakhani SR, International Agency for Research on Cancer (2012) WHO classification of tumours of the breast. World Health Organization classification of tumours, 4th edn. IARC, Lyon

16. Prat A, Perou CM (2011) Deconstructing the molecular portraits of breast cancer. Mol Oncol 5(1):5-23. https://doi.org/10.1016/j. molonc.2010.11.003

17. Hoadley KA, Yau C, Wolf DM, Cherniack AD, Tamborero D, Ng S, Leiserson MD, Niu B, McLellan MD, Uzunangelov V (2014) Multiplatform analysis of 12 cancer types reveals molecular classification within and across tissues of origin. Cell 158(4):929-944

18. Wolff AC, Hammond MEH, Allison KH, Harvey BE, Mangu PB, Bartlett JMS, Bilous M, Ellis IO, Fitzgibbons P, Hanna W, Jenkins RB, Press MF, Spears PA, Vance GH, Viale G, McShane LM, Dowsett M (2018) Human Epidermal growth factor receptor 2 testing in breast cancer: American Society of Clinical Oncology/College of American Pathologists Clinical Practice Guideline Focused Update. Arch Pathol Lab Med. https ://doi.org/10.5858/arpa.2018-0902-SA

19. McShane LM, Altman DG, Sauerbrei W, Taube SE, Gion M, Clark GM (2006) REporting recommendations for tumor MARKer prognostic studies (REMARK). Breast Cancer Res Treat 100(2):229-235. https://doi.org/10.1007/s1054 9-006-9242-8

20. Batistatou A, Televantou D, Bobos M, Eleftheraki AG, Kouvaras E, Chrisafi S, Koukoulis GK, Malamou-Mitsi V, Fountzilas GJ (2013) Evaluation of current prognostic and predictive markers in breast cancer: a validation study of tissue microarrays. Anticancer Res 33(5):2139-2145

21. Dekker TJ, Ter Borg S, Hooijer GK, Meijer SL, Wesseling J, Boers JE, Schuuring E, Bart J, van Gorp J, Mesker WEJBCR (2012) Determining sensitivity and specificity of HER2 testing in breast cancer using a tissue micro-array approach. Breast Cancer Res 14(3):R93

22. ERBB2 erb-b2 receptor tyrosine kinase 2 [ Homo sapiens (human)] (2019) NCBI (National Center for Biotechnology Information). https://www.ncbi.nlm.nih.gov/gene/2064. Accessed 24 June 2019

23. Marchiò $\mathrm{C}$, Lambros MB, Gugliotta $\mathrm{P}, \mathrm{Di}$ Cantogno LV, Botta $\mathrm{C}$, Pasini B, Tan DS, Mackay A, Fenwick K, Tamber N, Bussolati G, Ashworth A, Reis-Filho JS, Sapino A (2009) Does chromosome centromere copy number predict polysomy in breast cancer? A fluorescence in situ hybridization and microarray-based CGH analysis. J Pathol. https://doi.org/10.1002/path.2574

24. Goldhirsch A, Wood WC, Coates AS, Gelber RD, Thürlimann B, Senn H-J, Members P (2011) Strategies for subtypes-dealing with the diversity of breast cancer: highlights of the St Gallen International Expert Consensus on the primary therapy of early breast cancer 2011. Ann Oncol 22(8):1736-1747

25. Cheang MC, Chia SK, Voduc D, Gao D, Leung S, Snider J, Watson M, Davies S, Bernard PS, Parker JS (2009) Ki67 index, HER2 status, and prognosis of patients with luminal B breast cancer. J Natl Cancer Inst 101(10):736-750

26. Cheang MC, Voduc D, Bajdik C, Leung S, McKinney S, Chia SK, Perou CM, Nielsen TO (2008) Basal-like breast cancer defined by five biomarkers has superior prognostic value than triple-negative phenotype. Clin Cancer Res 14(5):1368-1376

27. Blows FM, Driver KE, Schmidt MK, Broeks A, Van Leeuwen FE, Wesseling J, Cheang MC, Gelmon K, Nielsen TO, Blomqvist C 
(2010) Subtyping of breast cancer by immunohistochemistry to investigate a relationship between subtype and short and long term survival: a collaborative analysis of data for 10,159 cases from 12 studies. PLoS Med 7(5):e1000279

28. Kim HK, Park KH, Kim Y, Park SE, Lee HS, Lim SW, Cho JH, Kim JY, Lee JE, Ahn JS, Im YH, Yu JH, Park YH (2018) Discordance of the PAM50 intrinsic subtypes compared with IHCbased surrogate in breast cancer patients: potential implication of genomic alterations of discordance. Cancer Res Treat. https://doi. org/10.4143/crt.2018.342

29. Nielsen TO, Parker JS, Leung S, Voduc D, Ebbert M, Vickery T, Davies SR, Snider J, Stijleman IJ, Reed J (2010) A comparison of PAM50 intrinsic subtyping with immunohistochemistry and clinical prognostic factors in tamoxifen-treated estrogen receptor-positive breast cancer. Clin Cancer Res 16:5222

30. Geiger T, Cox J, Mann M (2010) Proteomic changes resulting from gene copy number variations in cancer cells. PLoS Genet 6(9):e1001090
31. Fritz G, Brachetti C, Bahlmann F, Schmidt M, Kaina B (2002) Rho GTPases in human breast tumours: expression and mutation analyses and correlation with clinical parameters. Br J Cancer 87(6):635

32. Zhang B, Wang J, Wang X, Zhu J, Liu Q, Shi Z, Chambers MC, Zimmerman LJ, Shaddox KF, Kim S (2014) Proteogenomic characterization of human colon and rectal cancer. Nature 513(7518):382

Publisher's Note Springer Nature remains neutral with regard to jurisdictional claims in published maps and institutional affiliations. 